\title{
La médiation numérique et l'espace muséal en représentation : les portfolios vidéos d'agences de design prestataires de musées
}

Representing Digital Mediation and the Museum Space: Design Agencies'Video

Portfolios

\section{Ronan German}

\section{OpenEdition}

\section{Journals}

Édition électronique

URL : http://journals.openedition.org/edc/6509

DOI : 10.4000/edc.6509

ISSN : 2101-0366

\section{Éditeur}

Université Lille-3

\section{Édition imprimée}

Date de publication : 1 juin 2016

Pagination : $51-70$

ISBN : 978-2-917562-15-4

ISSN : $1270-684$

\section{Référence électronique}

Ronan German, «La médiation numérique et l'espace muséal en représentation : les portfolios vidéos d'agences de design prestataires de musées », Études de communication [En ligne], 46 | 2016, mis en ligne le 01 juin 2018, consulté le 05 mai 2019. URL : http://journals.openedition.org/edc/6509 ; DOI : 10.4000/edc. 6509 
La médiation numérique et l'espace muséal en représentation :

les portfolios vidéos d'agences de design prestataires de musées

Representing Digital Mediation and the Museum Space:

Design Agencies' Video Portfolios 
Cet article propose une analyse sémiologique de six vidéos réalisées par trois agences américaines (Second Story, Bluecadet et Local Projects) afin de valoriser des dispositifs numériques (tables et murs d'écrans tactiles) produits pour des musées dans le cadre de prestations. Ces vidéos reflètent, chacune à leur manière, le regard que posent ces agences sur le processus de médiation patrimoniale, sur le musée dans lequel il prend sens et sur ce que les conduites doivent être lors de la manipulation de ces dispositifs.

Mots-clés : analyse sémiologique, vidéo, agence, fantasmagorie, prétention, numérique.
Over the past few years, digital design agencies working with museums have systematically opted for the video medium to promote their productions. This article presents a semiotic analysis of six promotional videos produced by three American design agencies (Second Story, Bluecadet and Local Projects). Each video reflects, in its own way, how these agencies understand the process of heritage mediation and the museum space in which this process acquires meaning. The videos also reflect how the tools developed by these agencies should be used, thus creating an image of an ideal visitor experience.

Keywords: semiotic analysis, video, design agency, fantasy, communicational claim, digital media. 
Depuis le début des années 2010, une pratique semble s'être systématisée au sein d'agences de conception et de production de dispositifs de médiation numérique, prestataires de musées: la réalisation de courtes vidéos qui représentent ces dispositifs (tables et murs d'écrans tactiles, applications mobiles sur smartphones ou tablettes) en situation, insérés et manipulés dans l'espace d'exposition.

Cet article propose, dans une approche qui se veut exploratoire, une analyse sémiologique de six vidéos réalisées par trois agences américaines: Second Story ${ }^{1}$ (basée à Portland, Oregon), Bluecadet ${ }^{2}$ (basée à Philadelphie, Pennsylvanie) et Local Projects ${ }^{3}$ (basée à New York City). Ces agences ont conçu et réalisé des dispositifs pour des institutions telles que la Foundation for the National Archives (Washington, DC), le Penn Museum (Philadelphie, Pennsylvanie), ou encore, le Cleveland Museum of Art (Cleveland, Ohio).

Le choix de ces trois agences américaines se justifie par le fait que la réalisation de telles vidéos est quasi-inexistante sous cette forme dans les agences françaises qui réalisent le même type de prestations ${ }^{4}$. Les vidéos qui se rapprochent de ce type de production sont très majoritairement des vidéos institutionnelles produites par les établissements eux-mêmes pour rendre compte de l'insertion d'un dispositif dans leur panoplie d'aides à la visite ${ }^{5}$.

Le choix de ce corpus restreint s'est fait dans un double souci de repérer des vidéos qui présentent le même type de dispositif (à savoir, des tables et murs d'écrans tactiles) et qui cristallisent un ensemble de phénomènes et processus que nous souhaitions éclairer par cette analyse sémiologique dans l'article. Nous avons exploré les productions de plusieurs agences anglo-saxonnes (Pentagram, Wolff Olins, Sagmeister \& Walsh, Johnson Banks, Ziba, pour ne citer qu'elles) également prestataires de musées avant d'arrêter notre choix sur les trois agences américaines citées ci-dessus. Ainsi, six vidéos (deux par agences) forment notre corpus: I'espace Records of Rights dans l'exposition permanente de la Foundation for the National Archives ${ }^{6}$ et l'exposition permanente du National Civil Rights Museum ${ }^{7}$ (agence Second

1 http://secondstory.com/projects/browse/featured-work (consulté le19/01/16).

2 http://bluecadet.com/ (consulté le19/01/16).

3 http://localprojects.net/ (consulté le19/01/16).

4 Peut-être cette situation aura-t-elle évolué entre la rédaction de cet article et sa publication.

5 «Les jardins de Versailles, mode d'emploi!»: https://youtu.be/3Hx7wzcH97E (consulté le 09/09/2015).

6 http://secondstory.com/project/browse/featured-work/records-of-rights (consulté le19/01/16).

7 http://secondstory.com/project/browse/featured-work/ncrm (consulté le19/01/16). 
Story); I'exposition Native American Voices au Penn Museum ${ }^{8}$ et le Saint John Paul II National Shrine ${ }^{9}$ (agence Bluecadet); I'exposition permanente du Cooper Hewitt Smithsonian Design Museum ${ }^{10}$ et la Gallery One du Cleveland Museum of $\mathrm{Art}^{11}$ (agence Local Projects). L'analyse sémiologique de ces six vidéos s'est également portée sur l'environnement dans lequel elles s'inscrivent: les pages "projet» des sites internet et les articles des blogs des agences qui viennent compléter le discours - ou l'absence de discours - des vidéos.

Le parti pris adopté est celui d'une «interprétation»(Olivesi, 2006, 22) des intentions des auteurs de ces productions médiatiques uniquement à partir de ce qu'ils ont décidé de donner à voir dans ces vidéos. Malgré l'intérêt que présente le fait de donner la parole aux auteurs de ces productions pour confronter nos interprétations à leurs intentions, l'objectif de cet article ne réside pas dans cette confrontation.

Avant toute chose, il est important de préciser que ces agences ne réalisent pas ces vidéos dans le but qu'elles soient diffusées dans l'espace du musée ou qu'elles soient visionnées par des visiteurs. Ce ne sont pas eux les destinataires bien qu'ils en soient les principaux acteurs. Leur objectif est plutôt qu'elles soient visionnées par des responsables de musées qui, séduits par leur approche, convaincus de leurs compétences, pourraient faire appel à elles en tant que prestataires pour la conception et la réalisation de nouveaux dispositifs, comme ceux présentés dans ces vidéos.

Nous souhaitons insister, dans cet article, sur trois points en particulier. Tout d'abord, l'entremêlement de fils discursifs différents qui permettent trois lectures possibles de ces vidéos: promotionnelle, documentaire et institutionnelle. Ensuite, l'insertion de ces vidéos dans une fantasmagorie de l'utilisation de dispositifs numériques au musée. Enfin, la réalisation de ces vidéos comme moyen d'appuyer, de légitimer une prétention à intervenir sur le processus de médiation patrimoniale.

Ces trois points visent ainsi à éclairer ce que ces productions médiatiques disent du regard que semblent porter ces trois agences sur les usages de leurs dispositifs et sur les types de rapports au musée que ceux-ci suscitent et favorisent. 


\section{1. \\ Des vidéos susceptibles de plusieurs lectures}

Bien qu'elles adoptent des approches différentes pour valoriser le résultat de leur travail de conception et de production, les agences Second Story, Bluecadet et Local Projects réalisent toutes trois des vidéos susceptibles de trois lectures: promotionnelle (rendre compte de la qualité du produit pour séduire et convaincre de nouveaux clients), documentaire (justifier l'apport médiationnel du dispositif) et institutionnelle (insérer le discours du musée dans celui de l'agence).

\subsection{Faire la promotion du dispositif}

Le discours promotionnel qui vise à valoriser le dispositif produit par l'agence-prestataire au bénéfice du musée-client est présent autant dans les vidéos que dans l'environnement sémiotique dans lequel elles s'insèrent.

Les agences Local Projects et Second Story se décrivent avant tout comme des agences de design: "Local Projects est une entreprise de design de médias pour les musées et les espaces publics " $^{12}$; "Second Story est un réseau de studios de design ${ }^{13}$. Ainsi, ce qui ressort le plus souvent de ces vidéos sous leur aspect promotionnel, c'est la valorisation de compétences en matière de design.

Les vidéos et les textes qui les accompagnent sont l'occasion de montrer la qualité du dispositif produit et d'insister sur les savoir-faire de l'agence et sur le savoir-être de ses chefs de projet. Dans une vidéo à propos de l'exposition Treasures from Korea (Philadelphia Museum of Art), Marla Shoemaker, directrice du service éducatif parle de l'agence Bluecadet en ces termes: «Bluecadet s'est avéré être un partenaire idéal pour nous. Ils sont particulièrement bien informés sur la variété des possibilités d'interaction que proposent les écrans tactiles. C'était vif et stimulant, nous avons travaillé ensemble pendant deux ans pour produire ces choses et je dirais que ce fût un échange fructueux $»^{14}$. Le fait de donner le soin au musée-client de promouvoir le travail de l'agence en leur donnant la parole dans ces deux vidéos est particulièrement habile.

L'aspect promotionnel de ces vidéos est renforcé au sein des pages «projets » où la liste des compétences mobilisées est parfois couplée à une liste de nominations et de victoires à des prix de design ainsi qu'une liste d'articles de

12 "Local Projects is a media design firm for museums and public spaces».

13 "Second Story is a network of design studios".

14 «Bluecadet proved to be an ideal partner for us. They are quiteknowledgeable in a variety of possibilities of interactivitythattouchscreenspresent. It waslively and engaging, we workedtogether for about twoyears to producethese things and I would say it was a successful interaction ". 
presse qui mentionne les dispositifs réalisés. Par ailleurs, une agence comme Second Story a pris I'habitude de publier, à la fin d'un projet, un billet dans son blog qui éclaire le processus de production de tel ou tel dispositif afin de promouvoir autant le dispositif lui-même que les compétences des équipes projet.

Ainsi, la perspective promotionnelle des vidéos prend forme moins dans la construction d'un discours explicite qui vante les mérites et les compétences de l'agence (sauf dans les pages "projet») que dans les témoignages de satisfaction des responsables de musées-clients (chez Bluecadet), dans la valorisation des finitions en termes de design et, surtout, dans la captation et la mise en scène des comportements des visiteurs qui manipulent les dispositifs.

\subsection{Justifier l'apport médiationnel du dispositif}

Les agences ne peuvent pas se contenter de faire la seule promotion de leurs compétences dans ces vidéos. Il faut que des preuves tangibles, qui résultent de l'application de ces compétences par l'agence, soient clairement visibles. Dans ce cas, quel meilleur miroir pour justifier l'apport médiationnel d'un dispositif que celui de la satisfaction qui se lit directement sur les visages des visiteurs qui l'utilisent, le manipulent? Quel meilleur argument de réussite que leur enthousiasme?

Les agences prennent soin, lorsqu'elles développent un discours en ayant recours à une voix over, d'argumenter la façon dont leur dispositif s'insère dans l'espace en dialogue avec les autres "unités" (Davallon, 1999, 70-71) de l'exposition, avec les objets exposés et la façon dont il favorise certains rapports aux savoirs. Là aussi, l'agence Bluecadet laisse la parole à une représentante du Penn Museum, Kate Quinn (directrice du service des expositions et de la programmation), afin de présenter l'apport médiationnel de leur dispositif dans l'exposition Native American Voices.

Il est intéressant de relever que les trois agences élaborent trois façons très différentes de justifier l'apport médiationnel de leurs dispositifs dans leurs vidéos. L'agence Second Story a opté pour une voix over qui accompagne l'image. Le texte est entièrement écrit et narré par l'agence. Pour sa part, l'agence Bluecadet - bien qu'ayant également les compétences en interne-, délègue complètement la parole aux clients afin que ce soit eux qui justifient l'apport médiationnel de leurs dispositifs. L'agence ne prend ainsi jamais directement la parole dans ses vidéos, elle préfère simplement insérer quelques lignes de texte entre certaines scènes. Enfin, l'agence Local Projects a fait le choix de ne rien dire (aucune voix over, aucune délégation de parole aux responsables du musée-client) et de ne rien écrire (aucun texte ne vient accompagner les images). Leur parti pris est clair: l'apport médiationnel doit être une évidence. Néanmoins, là où les trois agences se retrouvent, c'est qu'à aucun moment elles ne donnent la voix aux visiteurs et à la façon dont ils appréhendent ces dispositifs. 


\subsection{Insérer le discours du musée dans celui de l'agence}

Si les trois agences adoptent trois approches discursives différentes quand il s'agit de justifier l'apport de leurs dispositifs en termes de médiation, elles adoptent par contre la même démarche lorsqu'il s'agit de renforcer leur propre discours par l'insertion d'éléments qui proviennent du discours institutionnel du musée-client.

Plus que d'apporter une caution de légitimité à leur discours, il s'agit davantage pour les agences de démontrer qu'elles ont bien respecté et intégré les valeurs de l'institution (mieux, qu'elles les partagent profondément) et qu'elles ont su, grâce à leur dispositif, faire siennes et répondre à ses préoccupations et problématiques.

Bien que cette démarche soit commune aux trois agences, l'insertion du discours du musée (à propos des valeurs qu'il défend, des missions qu'il assure, des projets qu'il conduit) dans le discours de l'agence ne va pas prendre la même forme. Second Story n'hésite pas, par exemple, à dédier une partie non négligeable de sa vidéo à des éléments qui ne montrent pas les dispositifs directement mais qui vont les insérer dans un projet d'établissement. Les vidéos pour le National Civil Rights Museum et l'exposition Records of Rights à la Foundation for the National Archives à Washington DC en sont de bons exemples. Ces deux vidéos dédient chacune une bonne dizaine de secondes (en début et en fin de vidéos) pour illustrer le cadre institutionnel dans lequel les dispositifs s'insèrent, accompagnés d'un discours sur les valeurs et missions de l'établissement que l'agence s'est appliquée à respecter.

National Civil Rights Museum: «Aujourd'hui, Le Lorraine Motel est un lieu de mémoire et de réflexion, une pierre de touche du passé qui relie les générations et demande aux visiteurs de prendre position pour un futur meilleur $»^{15}$.

15 «Today, the Lorraine Motel is a place for remembrance and reflection, a touch stone to the past that connects generations and asks visitors to take a stand for a better future». 


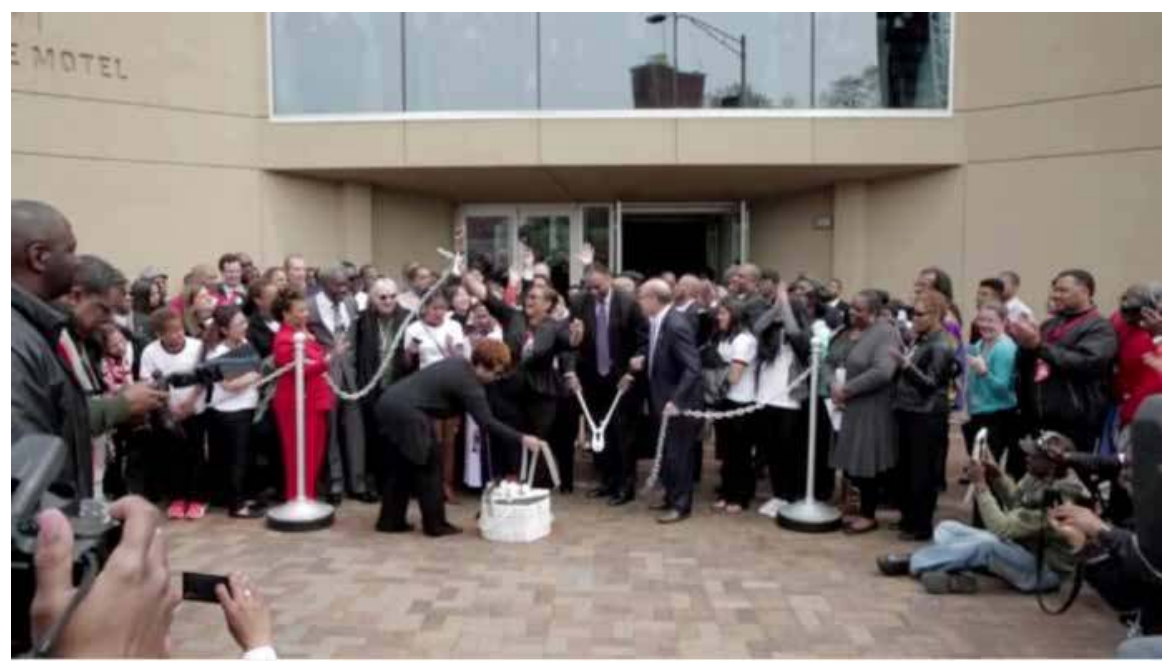

Figure 1: l'inauguration du musée national des droits civiques au Lorraine Motel (crédits : Second Story)

Ainsi, le discours de l'agence fait particulièrement écho au discours que I'on peut retrouver sur le site internet institutionnel. Par exemple: «Ceci est I'histoire de personnes, d'espoirs et de rêves, de défi et de changement. C'est une histoire américaine. Cette histoire et cette lutte, qui ont commencé il y a plusieurs siècles, continuent aujourd'hui- avec vous ${ }^{16}$.

De plus, les dispositifs de médiation développé en partenariat entre l'agence-prestataire et le musée-client servent d'appui à ce discours: pour le musée national des droits civiques, la principale table tactile de l'exposition consiste à mobiliser les visiteurs afin qu'ils s'engagent et "prennent position » (take a stand) à propos de la question des droits civiques. Dans l'exposition Records of Rights, la table tactile encourage les visiteurs à publiciser leur ressenti à propos de l'histoire des droits dans la société américaine par l'entremise de la diffusion de mots-clés sélectionnés par les visiteurs sur un mur d'écrans afin qu'ils soient lus par tous les visiteurs présents dans la pièce.

16 "This is the story of a people, of hopes and dreams, of challenge and change. It is an American story. This story and struggle that started many centuries ago, continues today- with you ". 


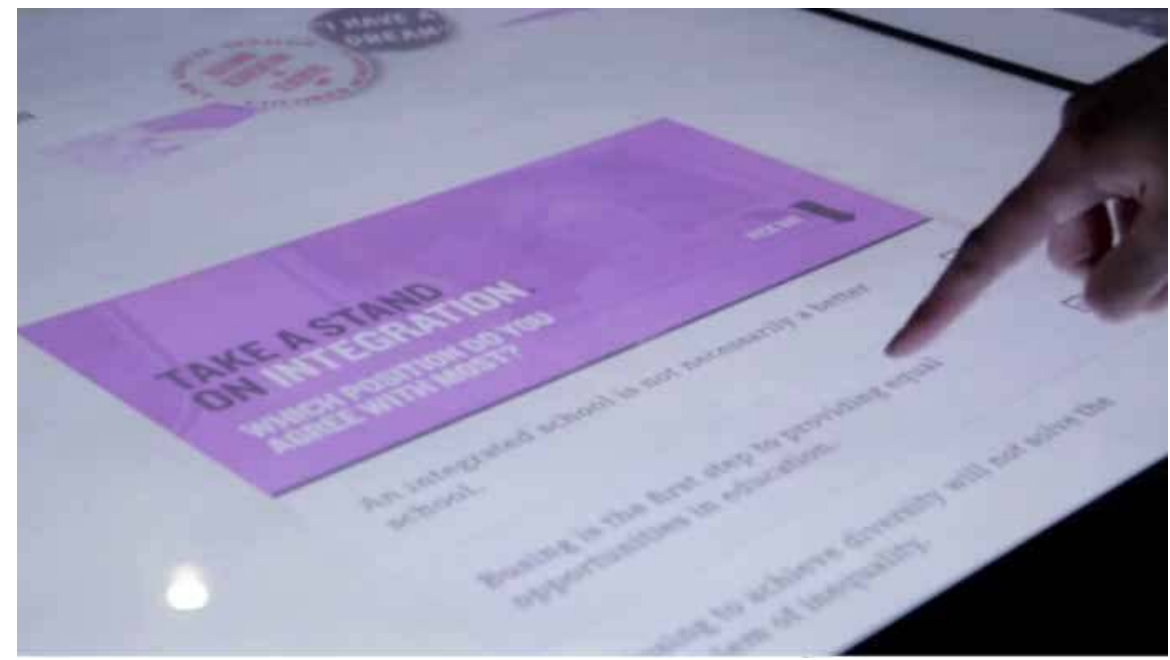

Figure 2: la table tactile enjoint les visiteurs à "prendre position» sur la question de l'intégration (National Civil Rights Museum) (crédits: Second Story)

On retrouve la même démarche dans les vidéos de l'agence Bluecadet qui, en donnant la parole aux responsables des musées-clients, permet à ces derniers de présenter leur établissement et/ou leur exposition et d'expliquer la façon dont l'agence a su faire siennes les valeurs de l'institution et les messages qu'elle vise à transmettre. C'est particulièrement le cas pour les vidéos qui présentent les dispositifs pour les expositions du Penn Museum (Native American Voices) et du Saint John Paulll National Shrine (table tactile « Journées mondiales de la jeunesse», World Youth Day).

Quant à l'agence Local Projects, celle-ci, nous l'avons vu, a fait le choix pour ses vidéos de n'utiliser ni voix over pour développer son propos, ni de donner la parole à des représentants de l'institution pour laquelle ils ont développé le dispositif. C'est dans la page "projet» de son site web que l'on peut repérer cette insertion d'éléments de discours du musée-client dans son propre discours ainsi que dans une sélection d'articles qui présentent leur travail dans la presse spécialisée ${ }^{17}$.

À l'issue de cette première partie, nous constatons que plusieurs fils discursifs s'entremêlent dans ces vidéos, complétés par les éléments que l'on peut trouver dans l'environnement sémiotique dans lequel elles s'insèrent. Jusque là, la démarche correspond tout à fait à ce qu'on attendrait de la part d'une agence qui cherche à valoriser les résultats de son travail. 
Ce que nous souhaitons faire maintenant, c'est insérer ces productions vidéo dans un ensemble plus large de dispositifs de représentation des pratiques, des «fantasmagories» (Jeanneret, 2014, 225-244).

\section{2. \\ Des vidéos qui alimentent une fantasmagorie de la visite au musée}

Tout d'abord, qu'entend-on nous par «fantasmagorie»? La fantasmagorie "prétend en effet agir sur les conduites, non en les conditionnant au sens technique, mais en exposant une multitude de représentations de ce qu'elles sont et doivent être et surtout en disséminant cette représentation à la manière d'un écosystème de perception, si l'on veut d'une panoplie phénoménologique, d'une phénopédie» (Jeanneret, 2014, 228).

Plusieurs chercheurs se sont intéressés au «pouvoir qu'ont ces images circulantes de produire une figuration et une implication de nos pratiques » (Jeanneret, 2014, 226). Ce qui intéressait Walter Benjamin (Benjamin, 2006), par exemple, c'était «la façon dont le dispositif fantasmagorique met en scène le corps et la façon dont l'image fantasmagorique convoque le corps regardant " (Jeanneret, 2014, 229). Ce qui intéresse une chercheuse comme Julia Bonaccorsi, dans ses «fantasmagories de l'écran » (Bonaccorsi, 2012), c'est de situer les productions médiatiques qui mettent en scène ces écrans «dans les espaces où elles font sens" (Jeanneret, 2014, 229).

Nous proposons ici d'appréhender les vidéos de ces agences (dont on pourrait qualifier de «fantasmagores» (Perriault, 1999, 125) les membres chargés de leur réalisation) comme autant de productions médiatiques qui s'insèrent dans une fantasmagorie de la manipulation de médias informatisés dans les institutions muséales. Que disent ces six vidéos des représentations des agences sur ce que "sont ou doivent être" les conduites des visiteurs lorsqu'ils manipulent leurs dispositifs?

\subsection{Un rapport attentif et plaisant aux savoirs}

Les vidéos oscillent sans cesse entre deux rapports aux savoirs (qui ne sont, bien sûr, pas antagonistes): d'une part, un rapport attentif et studieux, et d'autre part, un rapport plaisant et ludique voire créatif (au Cooper Hewitt Smithsonian Design Museum, par exemple).

On remarque rapidement dans les vidéos qu'une segmentation semble s'être opérée (consciemment ou non), notamment à partir de la variable de l'âge: on y voit, tour à tour, un adulte retraité, un adulte avec enfants, un jeune adulte en couple ou entre amis, un jeune adulte seul ou un jeune enfant accompagné de ses parents. La segmentation des visiteurs grâce à la variable 
de l'âge est très répandue (Eidelman et al., 2007; Mironer, 2001; Bourdieu et Darbel, 1966) bien qu'elle puisse être jugée insuffisante (Falk, 2009, 11, 27-28). À chacun de ces segments du public correspondrait donc un mode de rapport aux savoirs et aux dispositifs.

Dans la vidéo à propos du Saint John Paul II National Shrine (Bluecadet), on découvre les silhouettes de plusieurs visiteurs adultes de dos, éclairés par la lumière des écrans, en position statique ou se déplaçant avec des mouvements lents, adoptant une posture distante vis-à-vis des dispositifs.

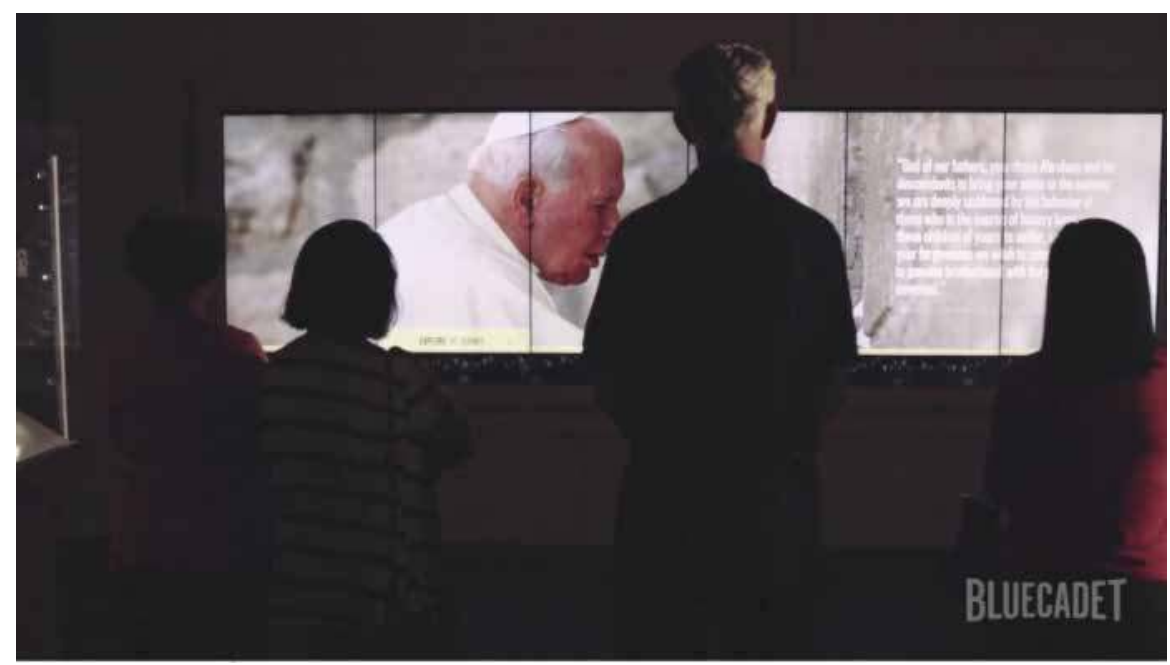

Figure 3: un groupe d'adultes regarde un mur d'écrans tactiles avant d'aller le manipuler (Saint John Paulll National Shrine) (crédits : Bluecadet)

Dans la vidéo qui présente les dispositifs du Cooper Hewitt Smithsonian Design Museum (Local Projects), les visiteurs se composent de jeunes adultes en couple ou entre amis et de jeunes enfants accompagnés de leur famille. Ici, le rapport aux savoirs est ludique, créatif, joyeux. On propose aux visiteurs, à l'accueil du musée, un stylo connecté qui leur permet d'utiliser les tables tactiles comme des tables de dessin afin de créer des modèles de mobilier, de tapisserie, etc. 


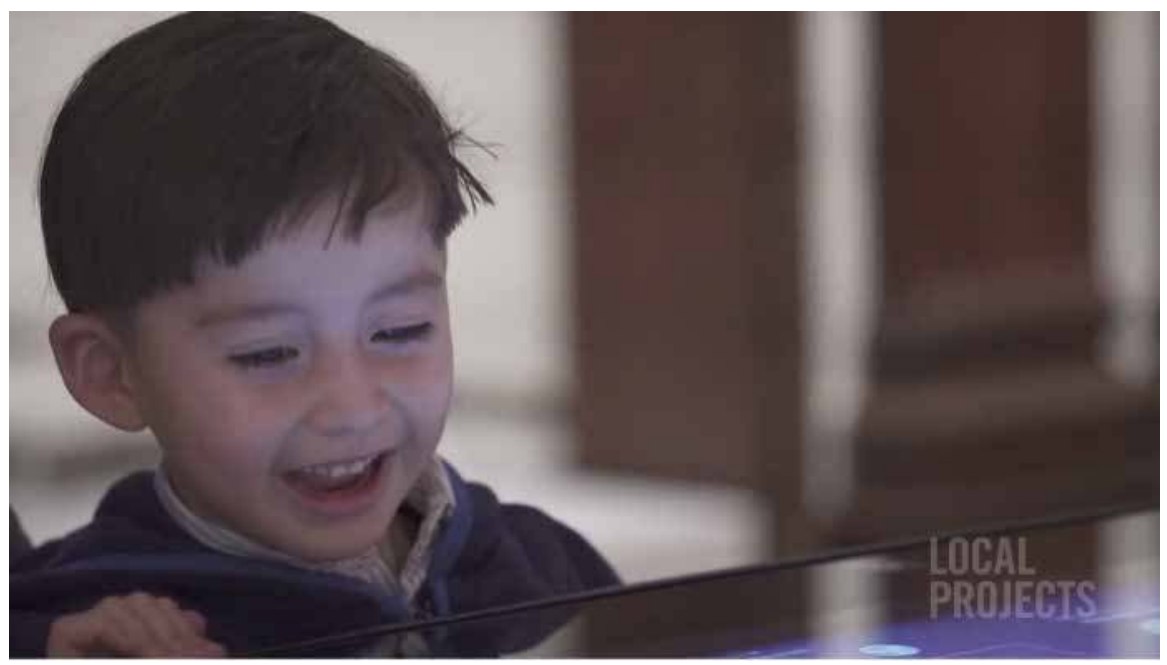

Figure 4: un enfant manipule une table tactile

(Cooper Hewitt National Design Museum) (crédits: Local Projects)

Tout au long de ces vidéos, force est de constater que deux types de publics apparaissent plus que d'autres parmi les visiteurs mis en scène: le public jeune et le public familial. Nous savons que le public jeune, par exemple, fait "l'objet d'un traitement souvent attentionné» (Chaumier et Mairesse, 2013, 123) de la part des institutions muséales. En mettant en scène ces publics particuliers dans leurs vidéos, les agences répondraient ainsi au souci des institutions de faire venir ces publics dans leurs établissements et valoriseraient leurs dispositifs comme un des facteurs principaux de ce succès. En cela, ces vidéos contribuent à renforcer, dans une fantasmagorie de la visite au musée, un rapport entre types de publics, types de médias et types de rapports aux objets/savoirs. Cependant, ce parallèle entre recours aux médias informatisés et augmentation de la fréquentation des institutions muséales de la part de ces publics particuliers est qualifié de «bancal »18 par les professionnels des musées eux-mêmes.

\subsection{L'exposition comme lieu d'interaction sociale}

Si une segmentation est opérée par les trois agences pour mieux mettre en scène et valoriser certaines postures, certains rapports au savoir, celles-ci se rejoignent sur le «rôle fondamental de l'interaction sociale au sein de l'expérience muséale» (Donnat et Tolila, 2003, 273). 
En effet, les vidéos d'agence valorisent et mettent très souvent en scène des moments d'échange et de complicité entre membres d'une même famille (Daignault et Schiele, 2014, 171-190; Eidelman et al., 2007, 147-159), entre amis ou entre partenaires d'un couple. On retrouve ces moments dans les vidéos de l'agence Second Story (autour de la table et du mur d'écrans tactiles du National Civil Rights Museum, autour de la table tactile de l'exposition Records of Rights), de l'agence Bluecadet (un adulte pointant son doigt vers un élément du mur d'écrans tactiles du Saint John Paul II National Shrine pour attirer l'attention de la personne qui l'accompagne) et de l'agence Local Projects (les jeunes adultes en train de dessiner sur une des tables tactiles, se prenant en photo devant leurs réalisations au Cooper Hewitt Smithsonian Design Museum, par exemple).

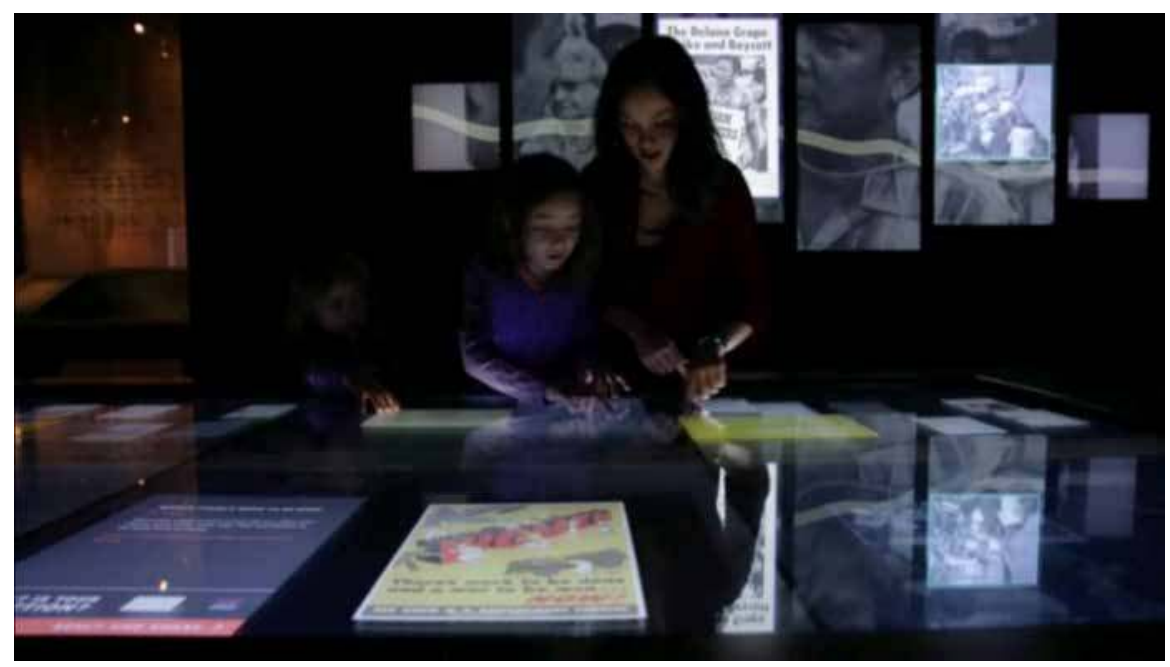

Figure 5: une femme et ses deux enfants manipulent la table tactile de l'exposition Records of Rights à la Foundation for the National Archives (crédits: Second Story)

Ainsi, ces (mises en) scènes méticuleusement filmées et montées, accompagnées d'une bande sonore savamment choisie illustrent bien la façon dont ces agences se représentent «ce que sont ou doivent être» les (bonnes) conduites à adopter lors de la manipulation et de la consultation de leurs dispositifs. Dans ces vidéos, aucun geste hésitant, aucune action sans résultat, aucun ralentissement, tout est assuré: le geste des visiteurs, la réactivité du dispositif, la solidité logicielle de l'application.

Comme nous l'avons rappelé en introduction, ces vidéos ne sont pas vouées à être visionnées par les visiteurs mais par les responsables de musées. Ce n'est donc pas aux premiers que ces représentations sont destinées mais bien aux seconds. Ainsi, il nous semble que ce qui fait l'efficacité de ces vidéos, c'est la façon dont elles parviennent à coupler la mise en scène des 
corps des visiteurs et la façon dont elles convoquent le corps regardant des responsables des musées. Le corps regardant de ces derniers est convoqué sous la forme d'un observateur invisible, fantomatique, qui flotte au-dessus et parmi les visiteurs.

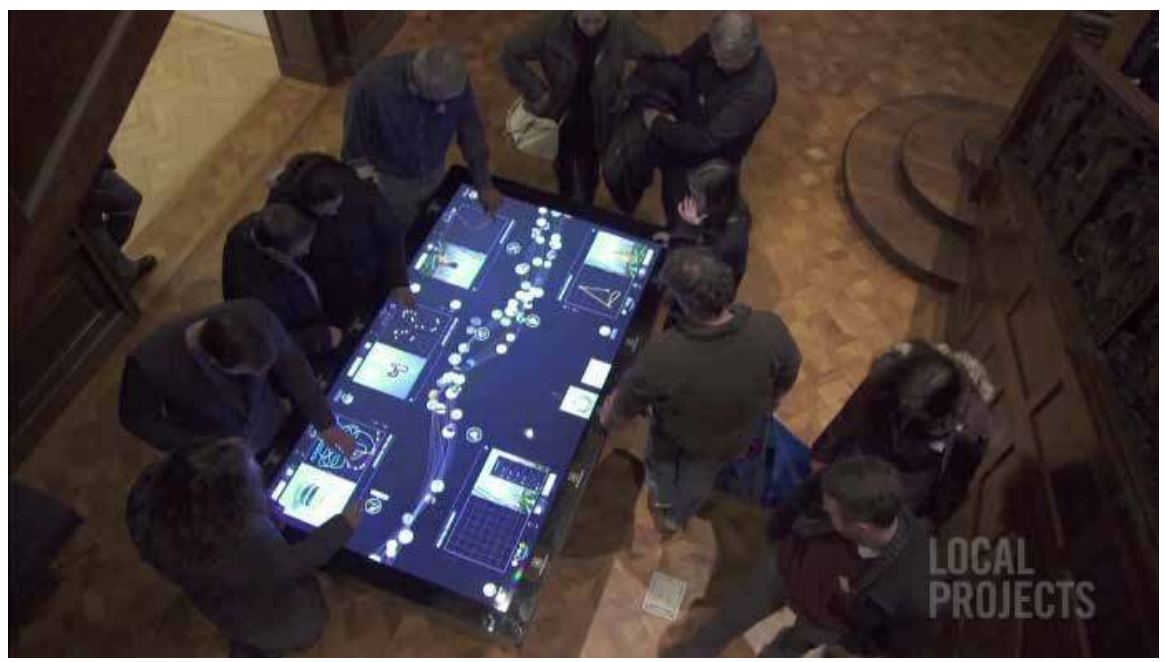

Figure 6: vue surplombante d'une table tactile au Cooper Hewitt National Design Museum (crédits : Local Projects)

On voit ainsi en quoi le déploiement d'un tel dispositif fantasmagorique semble pouvoir appuyer une prétention communicationnelle professionnalisée (Jeanneret, 2014, 256).

\section{3. \\ Les vidéos comme témoins d'une prétention communicationnelle}

Dans un glossaire en introduction de son ouvrage Critique de la trivialité, Yves Jeanneret définit ainsi la notion de prétention communicationnelle: «mode d'intervention sur les processus de communication qui de manière délibérée ou insensible hiérarchise les éléments, détermine les conditions cruciales et légitime une certaine compétence à y intervenir » (Jeanneret, 2014, 14).

Il nous semble que ces vidéos peuvent être appréhendées comme les témoins d'une prétention qui valorise un certain mode d'intervention sur le processus de communication qu'est la médiation patrimoniale. Ces vidéos peuvent également illustrer le résultat d'une "rencontre des prétentions" (Jeanneret, 2014, 247): celle d'une agence-prestataire et celle d'un musée- 
client qui n'interviennent pas de la même manière sur le processus de médiation patrimoniale. En témoigne, par exemple, l'absence complète, dans n'importe quelle vidéo, d'agents du musée chargés de la médiation humaine. Aucun agent de surveillance, aucun médiateur n'est présent dans ces vidéos. La seule présence visible d'un agent du musée dans ces six vidéos est celle d'un agent d'accueil qui distribue et explique le fonctionnement des stylos connectés à un couple qui vont leur permettre d'utiliser les tables tactiles du Cooper Hewitt National Design Museum. Consciemment ou non, ces vidéos semblent illustrer une prétention des agences à proposer un rapport au patrimoine qui ne nécessite aucune médiation humaine, seulement des dispositifs numériques.

Cependant, cela ne signifie pas que cette prétention professionnelle ne soit pas reconnue, voire même récompensée, par les acteurs des musées comme en témoigne les trois prix qu'un projet comme Gallery One, au Cleveland Museum of Art (Local Projects) a reçu lors de la conférence Museums and the Web en 2014 à Baltimore ${ }^{19}$.

\subsection{Susciter des pratiques valorisées}

Dans la partie précédente, nous avons décrit le travail de mise en scène des corps des visiteurs par les agences et la façon de convoquer le corps regardant des destinataires de ces vidéos. Il s'agit de compléter cette description par celle des formes sémiotiques qui suscitent les pratiques mises en scène. Dans toutes les vidéos réalisées par les agences, de nombreux plans se focalisent sur certains écrans. Que montrent-ils? Quelles pratiques suscitent-ils chez les visiteurs?

Plusieurs vidéos montrent des tables et écrans tactiles qui ont la particularité de rendre visibles, à toutes les personnes présentes dans l'espace où ils sont insérés, les actions entreprises par les visiteurs qui les manipulent. Il peut s'agir de prendre position sur un sujet («Aiderez-vous à combattre la pauvreté dans votre communauté? »20, National Civil Rights Museum, "Sélectionnez jusqu'à trois mots qui rendent compte de votre réaction pour les associez à l'image "21, Foundation for the National Archives), faire un choix dans une sélection d'objets numérisés («Sélectionner [cœur] pour sauvegarder des œuvres »22, Gallery One au Cleveland Museum of Art), se prêter à un exercice créatif «en direct» («Vous êtes en direct ${ }^{23}$, Cooper Hewitt National Design Museum) ou encore, laisser un message à un futur utilisateur sur une table tactile ("Le message que vous

19 http://mw2014.museumsandtheweb.com/best-of-the-web-winners/ (consultéle19/01/16)

20 "Will you help fight poverty in your community? ".

21 «Tap up to three reaction words to assign them to the image».

22 «Tap [heart] to save art works».

23 "You are live». 
avez sélectionné a été sauvegardé pour accueillir le prochain visiteur ${ }^{24}$, Saint John Paul II National Shrine).

Notons que, sans doute pour des raisons de modération des messages, aucun dispositif ne propose de texte libre. II s'agit soit d'une réponse par "oui » ou par «non» (National Civil Rights Museum), soit par la sélection de mots-clés (Records of Rights) ou de citations déjà figées (Saint John Paulll National Shrine).

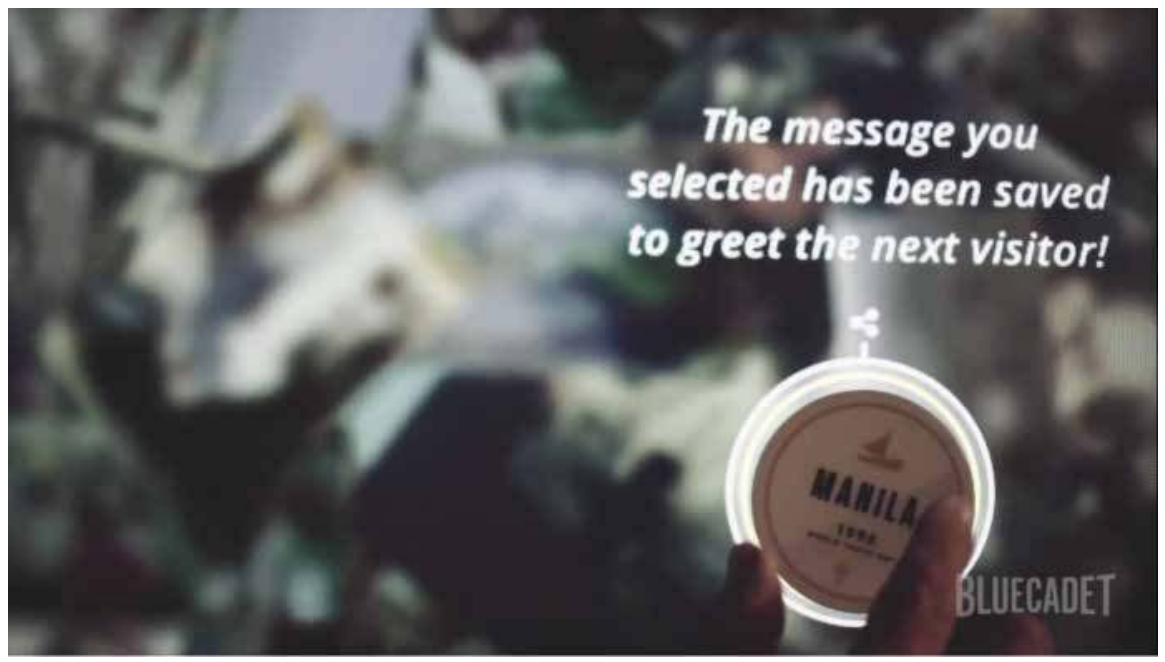

Figure 7: un visiteur peut choisir une citation du pape Jean Paulll pour accueillir le prochain visiteur sur la table tactile (Saint John Paulll National Shrine) (crédits : Bluecadet) 


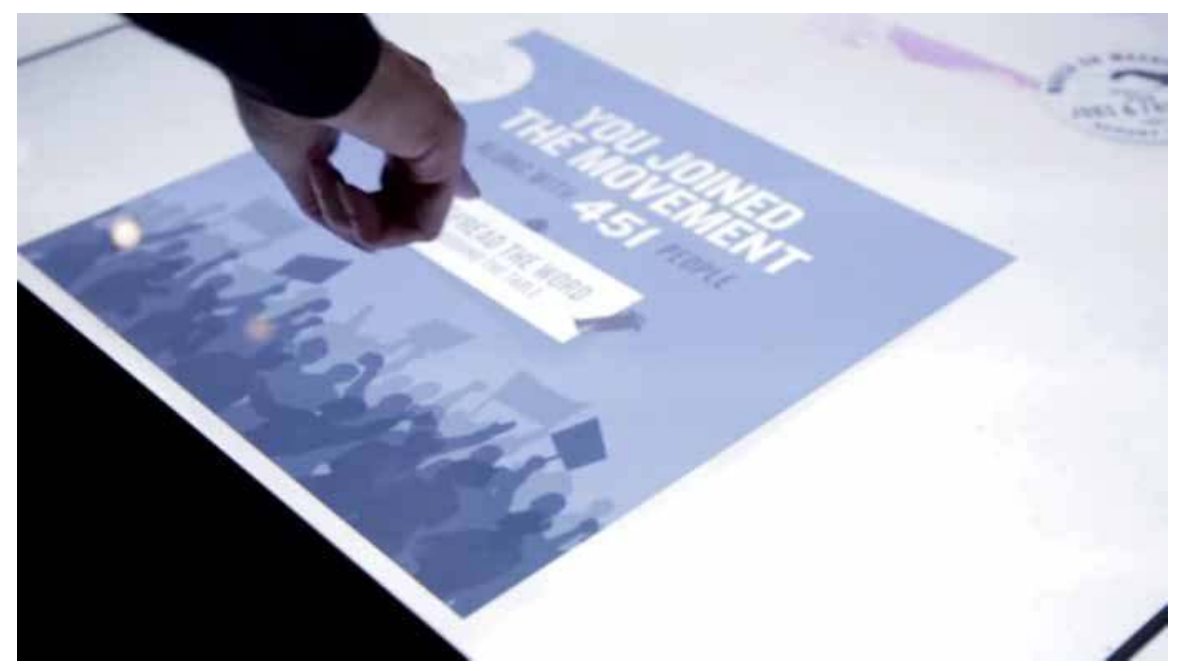

Figure 8: le visiteur peut rendre public sa prise de position («Portez la bonne parole ${ }^{25}$ ) et "rejoindre le mouvement » comme 451 autres personnes l'ont fait avant lui (National Civil Rights Museum) (crédits: Second Story)

Cette sélection d'écrans éclaire en partie la façon dont les agences s'y prennent pour susciter des pratiques valorisées lorsque le musée est appréhendé comme un lieu d'interaction sociale. D'ailleurs, cette représentation du musée est explicitement formulée par une des trois agences, Local Projects, dans la section "A propos» de son site internet: «Nous réinventons l'espace public grâce aux médias, en créant du sens et du lien dans des endroits aussi divers que les mémorials, à Times Square et dans les expositions d'art. Nous nous focalisons sur l'engagement des publics à travers les émotions et la technologie, en développant de nouvelles façons pour les gens d'interagir avec l'art, les villes et leurs semblables ${ }^{26}$.

Ainsi, une habile mise en scène des corps des visiteurs et une sélection précise des écrans à valoriser semblent permettre aux agences de formuler une prétention à intervenir dans le processus de médiation patrimoniale. Cependant, il est important de préciser que la formulation de cette prétention se nourrit aussi dans d'autres sphères.

25 «Spread the word».

$26 \mathrm{http}: / /$ localprojects.net/about/: «We're reinventing public space through media, creating meaning and connections in places as diverse as Memorials, Times Square, and art installations. We focus on engaging audiences through emotion and technology, developing new ways for people to interact with art, cities and one another» (Consulté le19/01/16). 


\subsection{Une prétention à l'intersection de plusieurs sphères}

Les trois agences n'ont pas seulement des musées pour clients. Elles réalisent des dispositifs semblables pour des entreprises comme Coca-Cola (Vault of the Secret Formula, Second Story, 2011) et Target (Target Open House, Local Projects, 2015) ainsi que pour le Financial Times (Graphic World Projection, Bluecadet, 2012). Une chercheuse comme Caroline Marti de Montety, dans ses analyses du processus de dépublicitarisation (Patrin-Leclère et al., 2014, 135141) a montré tout l'intérêt que portent les marques pour le média-exposition et son opérativité symbolique (Davallon, 1999).

Le mur d'écrans tactiles de l'espace Gallery One au Cleveland Museum of Art est intéressant à observer lorsque l'on appréhende les agences (Local Projects, dans ce cas) comme des vecteurs privilégiés pour le passage potentiel de formes médiatiques d'une sphère à l'autre. Dans cet espace, les visiteurs sont encouragés à "faire leur marché» dans la collection d'objets numérisés et présentés sur un mur de 12 mètres de long. Après avoir posé leur tablette sur un socle qui la connecte au mur d'écrans, les visiteurs sélectionnent les objets de collection qui les intéresse en les ajoutant à leur liste de favoris. Une fois cette sélection terminée, ils peuvent partir à la découverte des espaces d'exposition du musée.

Il ne s'agit aucunement de dénoncer la nature d'un dispositif comme celui-ci mais plutôt d'insister sur l'hybridation de formes médiatiques. Celle-ci est favorisée par les va-et-vient de ces agences d'une sphère à une autre, par la nécessité d'amortir des coûts liés à des développements spécifiques (utiliser une fonctionnalité dans un dispositif alors qu'elle a été développée pour un autre) ainsi que sur la capacité des médias informatisés (tels ces tables et murs d'écrans tactiles) à faire se rencontrer et cohabiter des formes sémiotiques aux origines diverses (le rayon de magasin, le site de e-commerce, la vitrine d'exposition, etc.) sur une même surface. Rappelons cependant que cette hybridation est un phénomène observé depuis longtemps dans les musées (Bennett, 1995).

\section{4. Conclusion}

Nous avons souhaité, dans cet article, attirer l'attention sur une forme médiatique originale qui ouvre une porte sur les représentations que ces agences prestataires de musées se font d'un processus de communication sur lequel elles interviennent, la médiation patrimoniale, et d'un espace dans lequel ce processus prend sens, le musée.

Nous avons souhaité ici concentrer notre analyse sur la façon dont plusieurs fils discursifs s'entremêlent au sein de ces vidéos, sur l'insertion particulière de ces vidéos dans une fantasmagorie de la visite au musée ainsi que 
sur l'appui qu'elles fournissent aux agences qui les réalisent pour formuler une certaine prétention.

Nous avons soulevé le fait que, dans ces vidéos, la mise en scène des corps des visiteurs semble aussi importante - voire plus importante-que la mise en valeur des dispositifs eux-mêmes. Ainsi, la sémiotisation d'un public idéal composé de «visiteurs-modèles" (Davallon, 1999, 15) apparaît comme une opération cruciale pour les agences. Ces vidéos visent à rendre désirable un public idéalisé (qui doit néanmoins rester crédible (Marin, 1981, 49-107) afin de convaincre les responsables de musées (les premiers destinataires de ces vidéos) de se munir de ce qui permet leur actualisation: les dispositifs produits par les agences.

Afin de compléter et d'approfondir cette première analyse, il nous semble intéressant d'élargir le spectre des productions médiatiques qui s'insèrent dans ces dispositifs de représentation des publics et de leurs pratiques afin de saisir les nombreux enjeux derrière leur sémiotisation. 
Bibliographie

Benjamin W. (2006). Paris, capitale du XIXe siècle. Le livre des passages, Paris, Éditions du Cerf.

Bennett T. (1995). The Birth of the Museum. History, theory, politics, London, Routledge.

Bonaccorsi J. (2012). Fantasmagories de l'écran, Mémoire pour l'HDR, Université Paris Sorbonne (Celsa).

ChaumierS. et MairesseF. (2013). La médiation culturelle, Paris, Armand Colin.

Daignault L. et SchieleB. (dir.). (2015). Les musées et leurs publics. Savoirs et enjeux, Québec, Presses universitaires du Québec.

Davallon J. (1999). L'Exposition à l'œuvre. Stratégie de communication et médiation symbolique, Paris, L'Harmattan.

Donnat O. et Tolila P. (dir.). (2003). Le(s) public(s) de la culture, Politiques publiques et équipements culturels, Paris, Presses de la fondation nationale des sciences politiques.

Eidelman J., Roustan M. et Goldstein B. (2007). La place des publics. De l'usage des études et recherches par les musées, Paris, La documentation française.
JeanneretY. (2014). Critique de la trivialité. Les médiations de la communication, enjeu de pouvoir, Paris, Éditions Non Standard.

Marin L. (1981). Le portrait du roi, Paris, Les Éditions de Minuit.

Patrin-LeclèreV., Marti de Montety C. et Berthelot-Guiet K. (2014). La fin de la publicité? Tours et détours de la dépublicitarisation, Paris, Le Bord de l'eau.

Perriault J. (1999). «Les 'fantasmagores'. De l'innovation dans les arts visuels». In MEI « Médiation et information», $n^{\circ} 10, p p .121-131$ 